\title{
Relaxation processes from charge-transfer excited states of organic radical 1,3,5-trithia- 2,4,6-triazapentalenyl crystals studied by ultrafast luminescence spectroscopy
}

\author{
Youtarou Takahashi* and Tohru Suemoto \\ Institute for Solid State Physics, The University of Tokyo, 5-1-5 Kashiwanoha, Kashiwa, Chiba 277-8581, Japan \\ Seiji Oguri and Jun Takeda \\ Department of Physics, Graduate School of Engineering, Yokohama National University, Yokohama 240-8501, Japan
}

(Received 16 December 2005; revised manuscript received 3 October 2006; published 15 November 2006)

\begin{abstract}
The excited state dynamics of 1,3,5-trithia-2,4,6-triazapentalenyl (TTTA) was investigated by using femtosecond luminescence spectroscopy. The luminescence was measured in low and high temperature phases of TTTA between 1.42 and $1.2 \mathrm{eV}$. Only in the low temperature phase, we observed a broad luminescence band with a decay time constant of $1.6 \mathrm{ps}$ in all energy range. This decay time constant suggests the existence of a large nonradiative relaxation path in the low temperature phase. Fast depolarization of the luminescence was also found, suggesting that an energy transfer between charge transfer excited states with different orientations are large and a few kinds of lattice distortion exist in this system.
\end{abstract}

DOI: 10.1103/PhysRevB.74.193104

The photoinduced phase transition (PIPT) has been attracting much attention and various kinds of materials which exhibit the PIPT were found recently. ${ }^{1-11}$ For understanding the PIPT phenomenon, the research on photoexcited states is important because of the photoinduced structural phase transition being induced by the cooperative rearrangement of the atomic positions in the excited states. As for 1,3,5-trithia2,4,6-triazapentalenyl (TTTA), the photoinduced magnetic and structural phase transition accompanied with disappearance of spin-Peierls ordering has been reported. ${ }^{9-11}$ The observed steady state luminescence with a Stokes shift suggests the existence of the excited state with lattice relaxation. ${ }^{11}$ Therefore, the investigation on metastable states with lattice relaxation and its dynamics by the time resolved luminescence spectroscopy is a first step to clarify the PIPT mechanism in TTTA. In this paper, we study the luminescence dynamics and its polarization characteristics, which provide information on the excited states and their relaxation processes.

The organic radical TTTA shows a magnetic phase transition with a large hysteresis loop over the temperature range from 230 to $305 \mathrm{~K} .{ }^{12}$ The high temperature (HT) phase consists of a uniform one-dimensional stacking of radicals and is paramagnetic, while the low temperature (LT) phase is diamagnetic because of strong dimerization of TTTA molecules along the stacking direction. ${ }^{12}$ The definition of the polarization refers to this stacking axis in the following. The charge transfer (CT) absorption bands locate at 1.8 and $2.1 \mathrm{eV}$ in the polarization parallel to the stacking axis and at $2.0 \mathrm{eV}$ in the polarization perpendicular to the stacking axis in the LT phase [see Fig. 2(b)]. ${ }^{13}$ In the HT phase, the absorption bands lie at 1.75 and $2.1 \mathrm{eV}$ in the polarization parallel and perpendicular to the axis, respectively. ${ }^{13}$ Under irradiation of visible laser pulse, the PIPT from the LT phase to the HT phase has been observed above the threshold excitation density of $10^{20}-10^{21}$ photons $/ \mathrm{cm}^{3}$ at room temperature. ${ }^{9-11}$

The HT phase of TTTA crystals were synthesized by the method described in Ref. 14. The LT phase crystals were prepared by cooling these HT phase crystals in a He gas flow cryostat at $100 \mathrm{~K}$. The time-resolved luminescence measurements were performed using the up-conversion technique.
PACS number(s): 78.47.+p, 71.35.-y

The samples were excited at photon energies of $1.82 \mathrm{eV}$ and $2.15 \mathrm{eV}$ from an optical parametric amplifier pumped by regenerative amplifier pulses and $1.55 \mathrm{eV}$ from fundamental laser pulses at a repetition rate of $200 \mathrm{kHz}$. These energies were fixed to the low and high energy CT absorption peaks for the polarization parallel to the stacking axis in the LT phase as shown in Fig. 2(b). The temperature was kept at $296 \mathrm{~K}$ or $250 \mathrm{~K}$ during the measurements, which are within the hysteresis loop. The average excitation power was $0.5 \mathrm{~mW}$ corresponding to the excitation density of 3 $\times 10^{17}$ photons $/ \mathrm{cm}^{3}$, which is smaller than the threshold density of the PIPT. The luminescence from the sample was collected by paraboloidal mirrors and mixed with the amplified fundamental pulses in a nonlinear crystal. Owing to the type I phase-matching condition, only one polarization component of the luminescence was converted into a sum frequency light. Then it was focused into a double grating monochromator and was detected by a photomultiplier tube (Hamamatsu Photonics R585) combined with a photon counter. For the polarization measurement we rotated the sample and the polarization of the excitation light at the same time. Therefore the detection sensitivity did not depend on the polarization of the luminescence. The polarization ambiguity originating due to the paraboloidal mirrors was order of $1 \%$. The time intervals of measurements were $33.3 \mathrm{fs}$ and the overall time resolution ranged from 90 to $130 \mathrm{fs}$, depending on the condition of the sample surface. The spectral response of the system was calibrated by using a tungsten standard lamp.

In steady state luminescence measurements, an Ar ion laser was used as an excitation source and the excitation photon energy was $2.41 \mathrm{eV}$. The luminescence was monochromatized by a single grating monochromator (Acton SpectraPro-500) and was detected by a cooled chargecoupled device (Princeton LN/CCD-1100PF).

Figure 1 shows the time dependence of the luminescence intensity in the LT and HT phases measured at $296 \mathrm{~K}$ under $1.82 \mathrm{eV}$ excitation. The sample temperature was kept at $296 \mathrm{~K}$, where both the high and low temperature phases are stable. The ordinate scale is proportional to the number of photons per unit energy interval per second. The background 


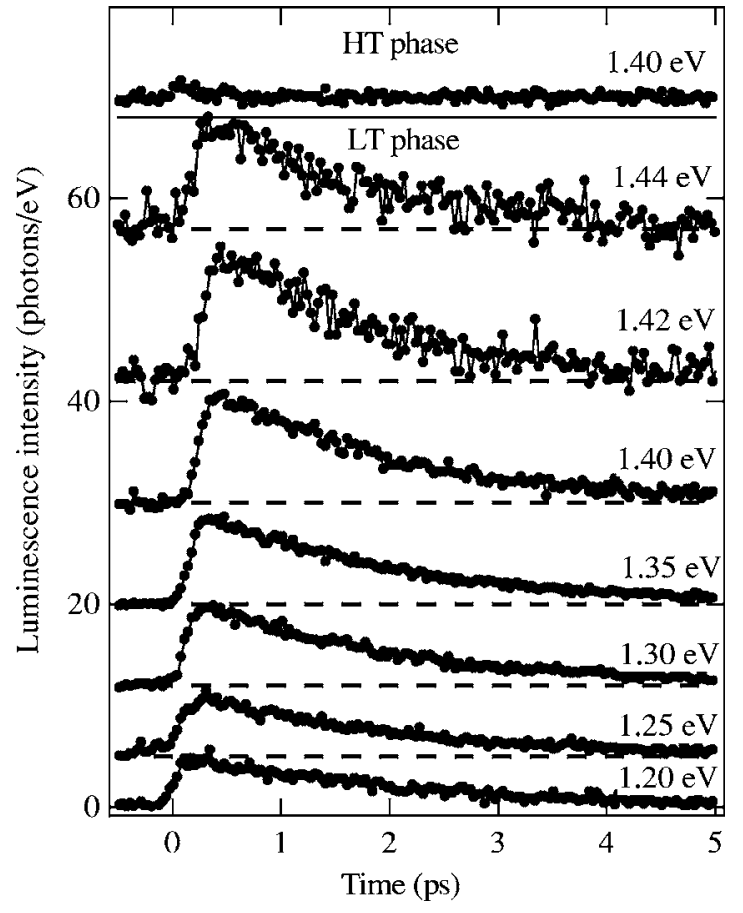

FIG. 1. The upper and lower curves are time evolution of the luminescence intensity in the HT phase and LT phase at $296 \mathrm{~K}$, respectively. The excitation light and luminescence were polarized parallel to the stacking axis [see Fig. 4(a)]. The luminescence photon energy is denoted right side near each curve. The ordinate scale is proportional to photon numbers per unit energy interval. The curves are shifted vertically for a convenient display; the dashed horizontal lines show the base line for each curve.

level was determined from the average intensity of the signal in an interval of 2 ps before the time origin and subtracted from the data. In the HT phase the lowest absorption peak assigned to the CT excitation lies at $1.75 \mathrm{eV}$ as shown in Fig. 2(b) and the pump pulses excited this band. ${ }^{13}$ As can be seen in Fig. 1, the decay profiles depend on the phase of TTTA. In the HT phase, only a small signal is found around the time origin, while in the LT phase a time evolution of luminescence is clearly observed. These results show that there is a large difference between the HT and the LT phases concerning the excited states and the relaxation processes. We observed luminescence from 1.2 to $1.44 \mathrm{eV}$ in the LT phase. Under excitation at higher photon energy of $2.15 \mathrm{eV}$, the time evolution of the luminescence in the LT phase crystal is similar to those observed in Fig. 1. Hereafter, we focus on the luminescence of the LT phase.

As shown in Fig. 2(a), the decay curve at $1.35 \mathrm{eV}$ can be fitted by a single exponential function with a decay time constant of $1.6 \mathrm{ps}$ in the LT phase. The decay time constant is the same in the energy range from 1.2 to $1.44 \mathrm{eV}$.

Shown in Fig. 2(b) are the luminescence spectrum in steady state (solid line) and the time-integrated luminescence intensity under 1.82 (open circles) and $2.15 \mathrm{eV}$ (triangles) excitations, obtained by integrating the luminescence signal from 0 to $5 \mathrm{ps}$ at a given luminescence photon energy. In up-conversion technique the second harmonic generation of $1.55 \mathrm{eV}$ laser source gives a large background as a stray light
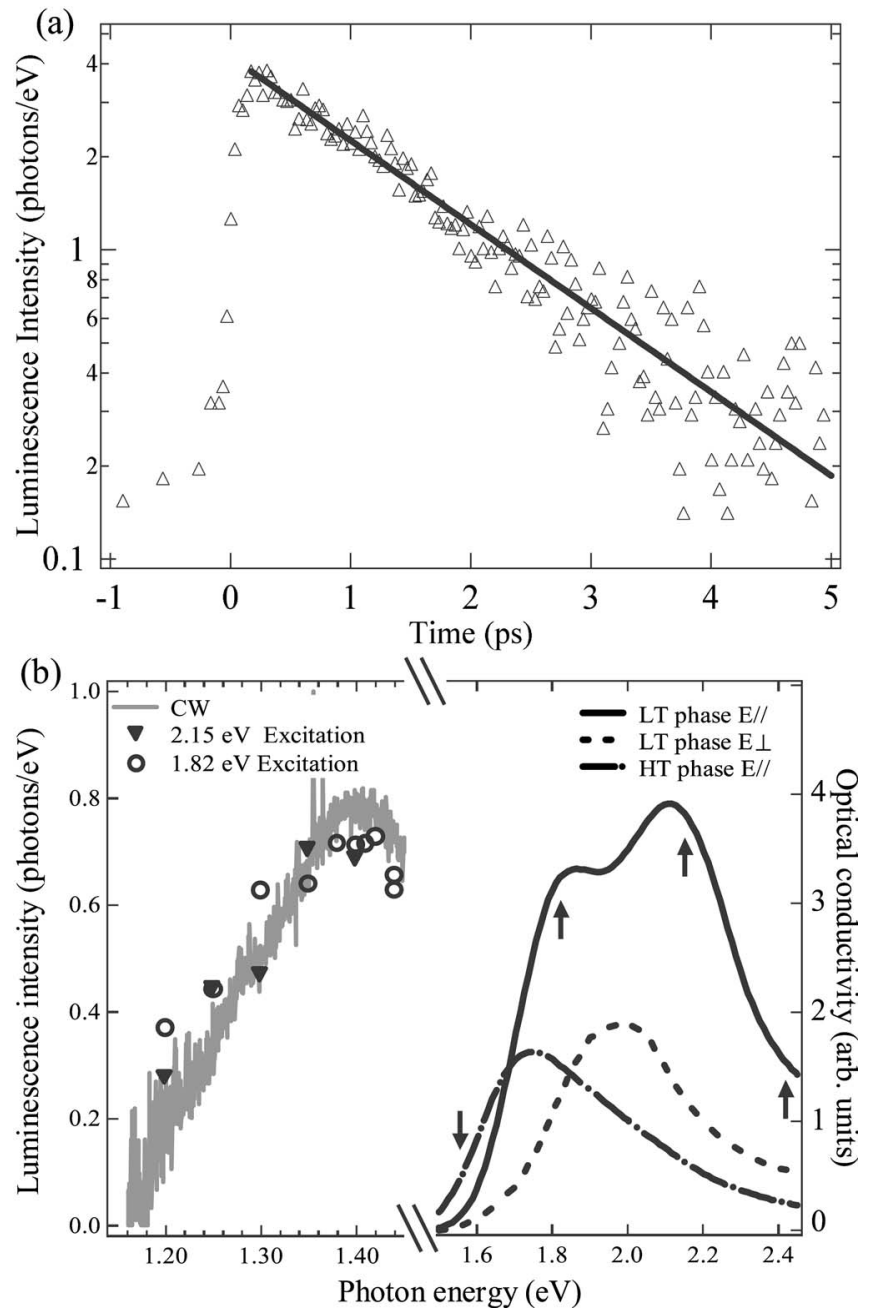

FIG. 2. (a) The open triangles show the time evolution of the luminescence intensity at $1.35 \mathrm{eV}$ under the room temperature of $296 \mathrm{~K}$ and solid line is a single exponential fitting function. (b) The gray line is a steady state luminescence spectrum in the LT phase at 296 K. The open circles show the integrated luminescence spectrum, which was constructed from the data on Fig. 1. The triangles show the integrated luminescence intensity under $2.15 \mathrm{eV}$ excitation light at $250 \mathrm{~K}$. The solid and dashed black lines are optical conductivity polarized parallel and perpendicular to the stacking axis in the LT phase, respectively. The dash-dot line shows the optical conductivity in the HT phase polarized parallel to the stacking axis. These optical conductivity spectra are reproduced from the Ref 13 . The arrows indicate the excitation photon energies (1.55, $1.82,2.15,2.41 \mathrm{eV})$.

in the monochromator. This background increases as the luminescence energy becomes close to $1.55 \mathrm{eV}$ and limits the observation of the high energy tail of the luminescence above $1.44 \mathrm{eV}$.

Since the integrated luminescence spectrum agrees with the steady state spectrum, the time evolution of the luminescence reflects the dynamics of that observed in steady state measurements. For the isolated TTTA molecules in dichloromethane solution, a luminescence band appears at $1.77 \mathrm{eV}$, which is far away from $1.4 \mathrm{eV}$. Defects or impurities are also unlikely to be the origin of the observed luminescence, be- 


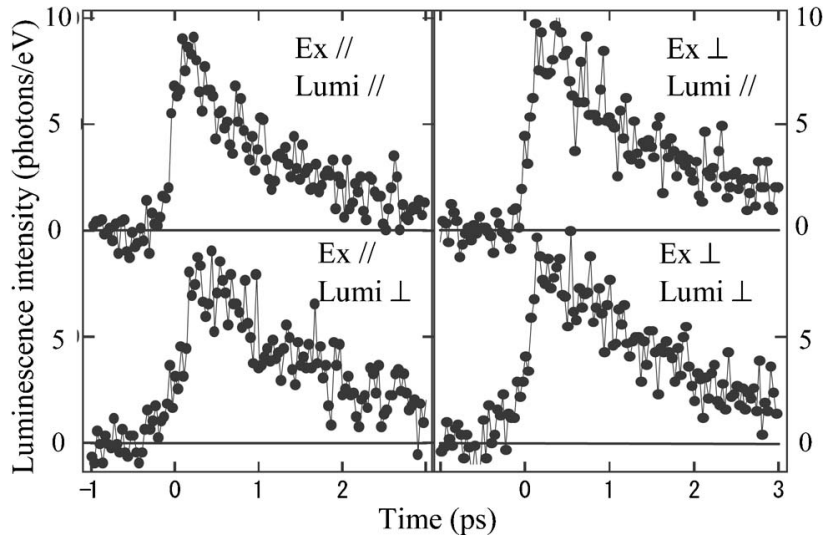

FIG. 3. The curves show the decay profiles of luminescence at $1.35 \mathrm{eV}$ in various combinations of luminescence and excitation polarizations. "Ex \|" and "Ex $\perp$ " indicate the excitation light polarization parallel and perpendicular to the stacking axis, respectively. Similarly, "Lumi \|" and "Lumi $\perp$ " show the luminescence polarization parallel and perpendicular to the stacking axis. The measurement temperature was $296 \mathrm{~K}$.

cause it rises up in a very short time $(<130 \mathrm{fs})$ after excitation of the CT band. Energy transfer process from the CT excited states to other optical centers would take a longer time because it involves electron transport and trapping. Therefore we conclude that the low energy luminescence is intrinsic for the LT phase crystal. The peak of the steady state luminescence is located at $1.4 \mathrm{eV}$ while that of the lowest CT absorption band lies at $1.8 \mathrm{eV}$, yielding a large Stokes shift of $0.4 \mathrm{eV}$, which indicates that a large lattice relaxation exists in the excited state of the LT phase. This suggests the existence of a self-trapped excitons (STEs) in the LT phase.

Since the expected radiative lifetime is usually longer than several nanoseconds in infrared region, the observed lifetime of $1.6 \mathrm{ps}$ is regarded as a nonradiative one. This indicates that almost all of the relaxed excited states return to the ground state through a nonradiative relaxation path in all polarization conditions.

Figure 3 shows the time dependence of the luminescence intensity measured at the photon energy of $1.35 \mathrm{eV}$ in different polarization configurations. The luminescence is completely depolarized within $130 \mathrm{fs}$ from the beginning of the excitation and does not depend on the polarization of the excitation light. This indicates that the excited states immediately lose the memory of the polarization of the excitation light.

Next, we consider the depolarized luminescence. The possible CT transitions and corresponding molecular rearrangement of the LT phase is schematically shown in Fig. 4(a). In this figure, the molecule pairs with a large overlap integral are shown. ${ }^{13}$ All the $\mathrm{CT}$ absorption bands in any polarization directions are excited by the $1.82 \mathrm{eV}$ photons, because each absorption band has a broad spectral width. For the polarization parallel to the stacking axis, there are two absorption bands located at 1.8 and $2.1 \mathrm{eV}$, which are assigned to the intradimer and interdimer $\mathrm{CT}$ transitions whose transition dipole is parallel to the direction of the column, respectively. ${ }^{13}$ For the polarization perpendicular to the stacking axis, on the
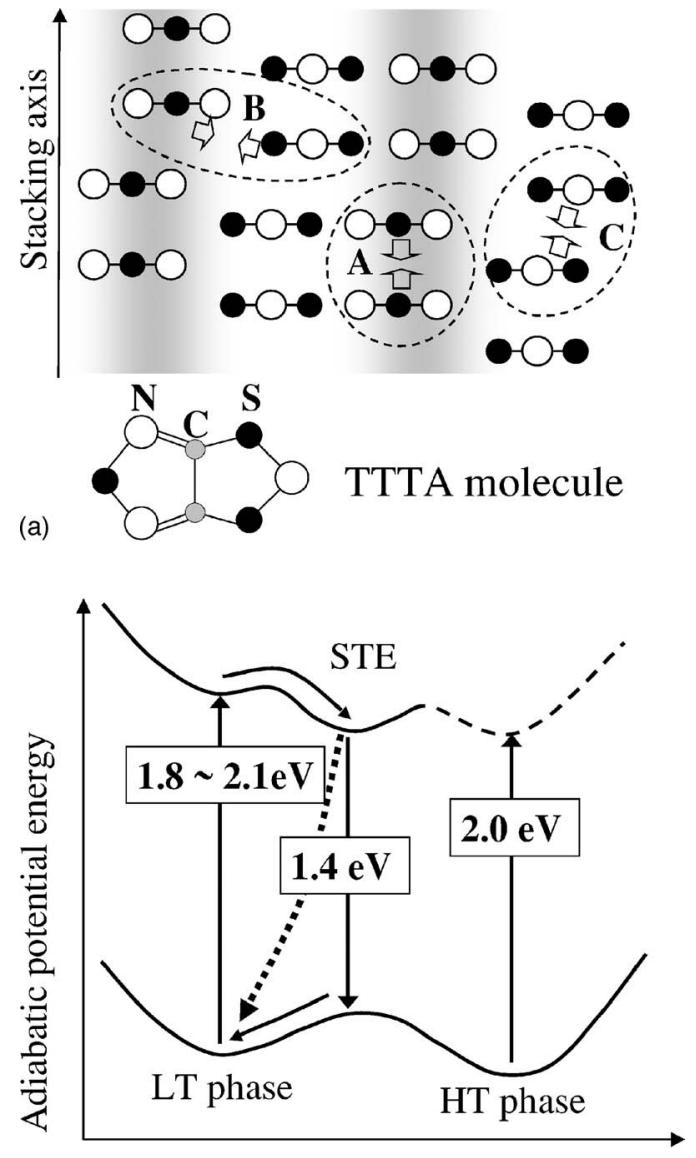

(b)

Order parameter

FIG. 4. (a) shows the schematic structure of the LT phase. The TTTA molecule structure is also represented. The identical side of the molecule is aligned within the stacking column and the side of the molecule is ordered alternately between the neighboring stacking columns. The $A, B$, and $C$ indicate pairs of TTTA molecules, in which the charge transfer takes place. The arrows show the direction of lattice distortion in three situations. (b) shows the adiabatic potential energy surfaces assumed in the model. The upward solid arrows indicate the excitation by photon and the downward solid arrow indicates the radiative relaxation. The downward dotted line corresponds to the nonradiative relaxation from STE.

other hand, there is an absorption band at $2.0 \mathrm{eV}$, which is assigned to the $\mathrm{CT}$ transition occurring between the stacking columns [see Fig. 2(b)]. ${ }^{13}$ Several configurations are possible for the CT excitons as shown by dashed ovals in Fig. 4(a) $A-C$. If the CT excited state is created along the stacking axis, the direction of the lattice relaxation will be the same as the direction of the CT transition, because TTTA molecules move along this direction due to the attractive Coulomb force as shown in Fig. 4(a) $A$ and $C$. On the other hand, the CT excitation perpendicular to the stacking axis will yield the lattice relaxation in the perpendicular direction as shown in Fig. 4(a) $B$. If the charges radiatively recombine on the same pair of molecules, the polarization direction should be conserved in luminescence. However, this is not the case in our experiment.

We propose a scenario for the depolarization processes of luminescence. Suppose $\tau_{\mathrm{tr}}$ as the time constant of the energy 
transfer between CT excitons in several dipole directions, $\tau_{\mathrm{STE}}$ as that of the STE generation, and $\tau_{\text {cool }}$ as that of the system cooling. As the CT bands having different dipole (polarization) directions overlap each other in energy, the energy transfer between the CT excited states will effectively take place. Since the TTTA crystal belongs to the triclinic system. ${ }^{13}$ The directions of the dipoles are not orthogonal. This makes the dipole energy transfer possible between the CT excitons with different orientation. In addition the electron transfer can occur between CT excitons with different dipole directions as shown by Fig. 4(a) $A-C$ and the existence of multiple transfer path will accelerate the depolarizing process. The $\tau_{\mathrm{STE}}$ correspond to the quarter of the lattice vibration period and its typical time constant is several tens or a hundred femtosecond. The cooling of the photoexcited states has been observed as a decreasing of the luminescence bandwidth for the STE in Pt complex and the typical time constants are several picoseconds. ${ }^{15}$ Here we can assume $\tau_{\text {tr }}<\tau_{\mathrm{STE}}<\tau_{\text {cool }}$. The laser pulses generate the CT excited states along the direction of its electric field. These CT excited states obey a Boltzmann distribution in high temperature $T_{\mathrm{el}}$ due to the excess energy and randomizations of the CT excitons occur within $\tau_{\text {tr }}$. Since $T_{\text {el }}$ can be larger than the energy difference between the CT exciton energies, the CT excitons in various directions will be uniformly distributed at the beginning. Then, the lattice relaxation takes place within $\tau_{\mathrm{STE}}$, and the STE state is created before cooling of the excited states. After the STE generation, the orientations of the dipoles are fixed and the luminescence is radiated. Finally, almost all of the STE annihilate through the nonradiative relaxation path with a lifetime of $1.6 \mathrm{ps}$. This scheme enables us to interpret the completely depolarized luminescence with a large Stokes shift. The luminescence intensity depends on the oscillator strength and we can estimate that of STE by the polarized absorption spectrum. The intensity of the absorption peak polarized perpendicular to the stacking axis is about one half of that polarized parallel to the stacking axis as shown in Fig. 2(b) indicating that the oscillator strength for both directions are in the same order. The relaxed excited states, i.e., STE, in each polarization will have the same or- der of oscillator strength, because the lattice distortion will not change the oscillator strength very much in each polarization. Thus the same intensity of the luminescence in each polarization suggests the same order of distribution of STE.

In the case of low excess energy, namely $1.55 \mathrm{eV}$ excitation near the band edge, the luminescence intensity in each polarization was not the same. It was found that the luminescence intensity polarized parallel to the stacking axis was approximately two times of that polarized perpendicular to the stacking axis in either excitation polarization conditions. This result is consistent with the band edge lineup of the CT excitons parallel and perpendicular to the stacking axis.

The proposed adiabatic potential energy surfaces in the LT and HT phases at room temperature are schematically shown in Fig. 4(b). At room temperature, the potential minimum of the LT phase is higher than that of the HT phase in the ground state, because this temperature is very close to the upper edge of the hysteresis loop. In the excited state, a potential minimum corresponding to the STE state is located slightly below the CT excited state. The excited states generated in the LT phase relax to the potential minimum of the STE and finally almost all of them relax to the ground state through a nonradiative relaxation path within $1.6 \mathrm{ps}$ (dashed arrow).

In conclusion, we investigated the excited state dynamics of TTTA crystal through the luminescence and its depolarization in femtosecond regime. In the LT phase, the timeevolution of luminescence with a time constant of $1.6 \mathrm{ps}$ was observed. This time constant implies that a nonradiative relaxation is dominant as the relaxation path. From the fast depolarization of the luminescence, it is strongly suggested that the excited state dipole is depolarized immediately after the excitation through the energy transfer between CT excited states in various directions and then a few kinds of lattice relaxations take place in the LT phase of TTTA. It is desired to clarify the lattice distortion mode of STE and its dynamics.

This work was supported in part by the Grant-in-Aid for Scientific Researches (A) and (B) from the Ministry of Education, Culture, Sports, Science, and Technology of Japan.

\footnotetext{
*Electronic address: youtarou@issp.u-tokyo.ac.jp

${ }^{1}$ K. Nasu, in Relaxations of Excited States and Photoinduced Structural Phase Transitions, Springer Series in Solid State Science, Vol. 124 (Springer, Berlin, 1997).

${ }^{2}$ K. Nasu, in Photoinduced Phase Transitions (World Scientific, Singapore, 2004).

${ }^{3}$ S. Koshihara, Y. Tokura, K. Takeda, and T. Koda, Phys. Rev. Lett. 68, 1148 (1992).

${ }^{4}$ K. Tanimura and I. Akimoto, Phase Transitions 75, 715 (2002).

${ }^{5}$ S. Iwai, S. Tanaka, K. Fujinuma, H. Kishida, H. Okamoto, and Y. Tokura, Phys. Rev. Lett. 88, 057402 (2002).

${ }^{6}$ Y. Ogawa, S. Koshihara, K. Koshino, T. Ogawa, C. Urano, and H. Takagi, Phys. Rev. Lett. 84, 3181 (2000).

${ }^{7}$ T. Tayagaki and K. Tanaka, Phys. Rev. Lett. 86, 2886 (2001).

${ }^{8}$ M. Fiebig, K. Miyano, Y. Tomioka, and Y. Tokura, Science 280,
}

1925 (1998).

${ }^{9}$ J. Takeda, M. Imae, O. Hanado, S. Kurita, M. Furuya, K. Ohno, and T. Kodaira, Chem. Phys. Lett. 378, 456 (2003).

${ }^{10}$ S. Oguri, O. Hanado, J. Takeda, M. Furuya, K. Ohno, S. Inoue, and T. Kodaira, J. Lumin. 112, 283 (2005).

${ }^{11}$ H. Matsuzaki, W. Fujita, K. Awaga, and H. Okamoto, Phys. Rev. Lett. 91, 017403 (2003).

${ }^{12}$ W. Fujita and K. Awaga, Science 286, 261 (1999).

${ }^{13}$ W. Fujita, K. Awaga, H. Matsuzaki, and H. Okamoto, Phys. Rev. B 65, 064434 (2002).

${ }^{14}$ G. Wolmershäuser and R. Johann, Angew. Chem., Int. Ed. Engl. 28, 920 (1989).

${ }^{15}$ T. Matsuoka, J. Takeda, S. Kurita, and T. Suemoto, Phys. Rev. Lett. 91, 247402 (2003). 